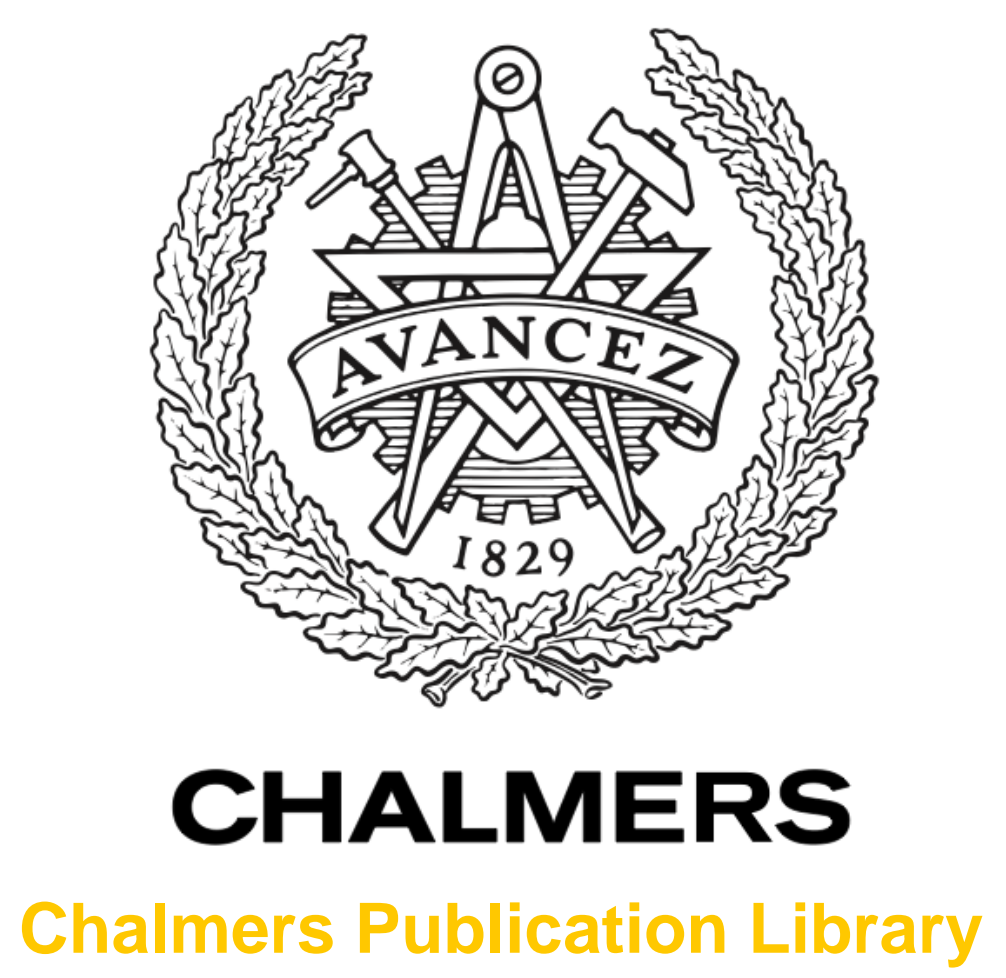

\title{
A study of uncertainty models in a reverberation chamber at NIST
}

This document has been downloaded from Chalmers Publication Library (CPL). It is the author's version of a work that was accepted for publication in:

2012 IEEE International Symposium on Antennas and Propagation, Chicago, USA, July 814, 2012 (ISSN: 1522-3965)

Citation for the published paper:

Engvall, E. ; Holloway, C. ; Ladbury, J. (2012) "A study of uncertainty models in a reverberation chamber at NIST". 2012 IEEE International Symposium on Antennas and

Propagation, Chicago, USA, July 8-14, 2012

http://dx.doi.org/10.1109/APS.2012.6349058

Downloaded from: http://publications.lib.chalmers.se/publication/163384

Notice: Changes introduced as a result of publishing processes such as copy-editing and formatting may not be reflected in this document. For a definitive version of this work, please refer to the published source. Please note that access to the published version might require a subscription.

Chalmers Publication Library (CPL) offers the possibility of retrieving research publications produced at Chalmers University of Technology. It covers all types of publications: articles, dissertations, licentiate theses, masters theses, conference papers, reports etc. Since 2006 it is the official tool for Chalmers official publication statistics. To ensure that Chalmers research results are disseminated as widely as possible, an Open Access Policy has been adopted.

The CPL service is administrated and maintained by Chalmers Library. 


\section{A Study of Uncertainty Models in a Reverberation Chamber at NIST}

\author{
Erik Engvall, Christopher L. Holloway, John M. Ladbury \\ Electromagnetics division \\ National Institute of Standards and Technology (NIST) \\ Boulder, Colorado, United States \\ karl.engvall@nist.gov
}

\author{
Per-Simon Kildal \\ Antenna group, Signals and System \\ Chalmers University of Technology \\ Gothenburg, Sweden
}

\begin{abstract}
The reverberation chamber has become a good alternative for over-the-air (OTA) testing of small antennas. Here the uncertainty of total radiated power measurements is evaluated for a reverberation chamber at National Institute of Standards and Technology (NIST), Boulder, Colorado. A procedure for measuring the uncertainty based on the standard deviation of nine different antenna orientations in the RC has been used.

A model for the uncertainty, developed at Chalmers University of Technology, has been evaluated for measurements in this chamber. The model is based on the standard deviation of a Gaussian distribution for both the non-line-of-sight and the line-of-sight components together with an average Rician K-factor.

It is found that the agreement between theoretical and experimental values are good and that the model describes the uncertainty well for both various loading in the chamber and various amount of platform stirring.
\end{abstract}

\section{INTRODUCTION}

Traditionally the reverberation chamber $(\mathrm{RC})$ has been used for EMC testing of electrical devices, but it has the last twelve years been developed into a test facility for characterizing small antennas [1]. It can be used for measuring radiation efficiency, total radiated power, diversity gain, maximum available apacity of MIMO systems, receiver sensitivity and data throughput [2]. The theory of reverberation chambers is well understood [3], as well as the uncertainty by which received power can be estimated [4]. An improved model for uncertainty has been assessed in [5]. The formulation is described in detail in [6], and is based on a model where the uncertainty is caused by a Line-Of-Sight (LOS) contribution in the chamber and characterized by an average K-factor [7]. The model is in [5] and [6] compared with experimental values from a Bluetest reverberation chamber at Chalmers, also used to demonstrate good uncertainty by comparison with measurement in anechoic chambers [8]. The subject of this paper is to validate the uncertainty model with experimental measurements in a larger reverberation chamber at National Institute of Standards and Technology (NIST).

\section{Theory for Uncertainty Model and Average RICIAN K-FACTOR}

It can be argued that the the S-parameters $S_{11}$ and $S_{21}$ can be divided into two parts, one deterministic contribution and one statistic contribution. The deterministic contribution will be the same as a free space contribution, a line-of-sight (LOS) contribution. The statistic contribution comes from the stirred fields in the reverberation chamber, a non-line-of-sight (NLOS) contribution. The uncertainty in an average power estimate based on only the chamber contribution (the NLOS part) is a traditional relative Gaussian standard deviation [4]. In terms of the number of independent samples, $N_{\text {ind }}$, the standard deviation becomes as in (1). $N_{\text {ind }}$ is the product of number of independent plate and platform positions and number of chamber antennas used.

$$
\sigma_{N L O S}=1 / \sqrt{N_{\text {ind }}} \quad N_{\text {ind }}=M_{\text {plate }} M_{p f} M_{\text {ant }}
$$

The deterministic contribution from the LOS is governed by Friis' equation. The contribution is proportional to the radiation efficiency if the antennas are oriented arbitrary. This is often the case with a normal user, and also in the case with platform stirring. The standard deviation of this estimation of efficiency will again follow a Gaussian distribution and have the relation in (2). $N_{L O S, i n d}$ is the product of number of platform positions and number of wall antennas that changes the angle of arrival so much that they can be regarded as different contributions.

$$
\sigma_{L O S}=1 / \sqrt{N_{L O S, \text { ind }}} \quad N_{L O S, \text { ind }}=M_{p f} M_{\text {ant }}
$$

The final formula for the RC uncertainty in an average power estimate when accounting for the average K-factor is expressed in (3) [5]. The standard deviation has been weighted with the average $\mathrm{K}$-factor, $K_{a v}$, to account for the relative distribution of the two contributions.

$$
\sigma=\sqrt{\left(\sigma_{N L O S}\right)^{2}+K_{a v}\left(\sigma_{L O S}\right)^{2}} / \sqrt{1+K_{a v}^{2}}
$$

The Rician K-factor is a measure of the amount of direct coupling in the chamber relative to the multipath component provided by the chamber. The K-factor varies with position in the chamber, therefore it is convenient to use an 
averaged $K_{a v}$ over all positions of the platform as defined in [7].

$$
K_{a v}=\sum P_{L O S} / \sum P_{N L O S}
$$

The parts in (4) are the same as for a normal Kfactor calculation: the amount of unstirred energy (the LOS contribution) related to the stirred energy (NLOS contribution). These can be calculated via the measured S-parameters as [7], i.e,

$$
P_{L O S}=\left|\overline{S_{21}}\right|^{2} \quad P_{N L O S}=\overline{\left|S_{21}-\overline{S_{21}}\right|^{2}}
$$

The bars in (5) represents the mean over all plate stirrer positions, so that $P_{N L O S}$ becomes the variance of $S_{21}$.

\section{Results And Conclusion}

The RC at NIST has dimensions $4.3 \mathrm{~m} \times 3.8 \mathrm{~m} \times 2.9 \mathrm{~m}$. The stirring is performed by two rotational paddles, one vertical and one horizontal.

To provide platform stirring the reference antenna was placed on a positioner able to move in the $\mathrm{x}$-y-plane, thus the reference antenna will point in the same direction for all points on the positioner.

To measure the uncertainty nine measurements of the average power transfer function was made. The nine measurements represents three different orientations of the reference antenna (vertical, tilted 45 degrees and horizontal) at three different heights. The standard deviation around the mean of these measured power transfer functions was taken as a measure of the uncertainty of the chamber.

The reference antenna used was a wideband $(650 \mathrm{MHz}-$ $3 \mathrm{GHz}$ ) discone antenna. As wall antenna a dual ridge horn, pointed towards one of the stirrers, was used.

Relative total standard deviation from measurements and the uncertainty model are compared in Fig. 1. The results are presented in $\mathrm{dB}$. The dashed curves represents the theoretical values from the model and the solid curves are experimental results. Both obtained by using one wall antenna, nine platform positions and 100 unique stirrer configurations.

The model works fine up to about $2 \mathrm{GHz}$ where a discrepancy occurs. This might be due to correlation issues while the platform is not rotating so that the antenna points in the same direction for all measurements.

A verification of the improved uncertainty model for a new measurement environment has been presented. The model coincide well with experimental values for the reverberation chamber at NIST and together with the results in [5] this is a strong indicator for the validity of the model. The model can describe the uncertainty both with variations in loading and in amount of platform stirring.

\section{REFERENCES}

[1] K. Rosengren, P.-S. Kildal C. Carlsson and J. Carlsson, "Characterization of Antennas for Mobile and Wireless Terminals in Reverberation Chambers: Improved Accuracy by Platform Stirring", Microwave and Optical Technology Letters, vol. 30, no. 20, pp. 391-397, Sept. 2002.
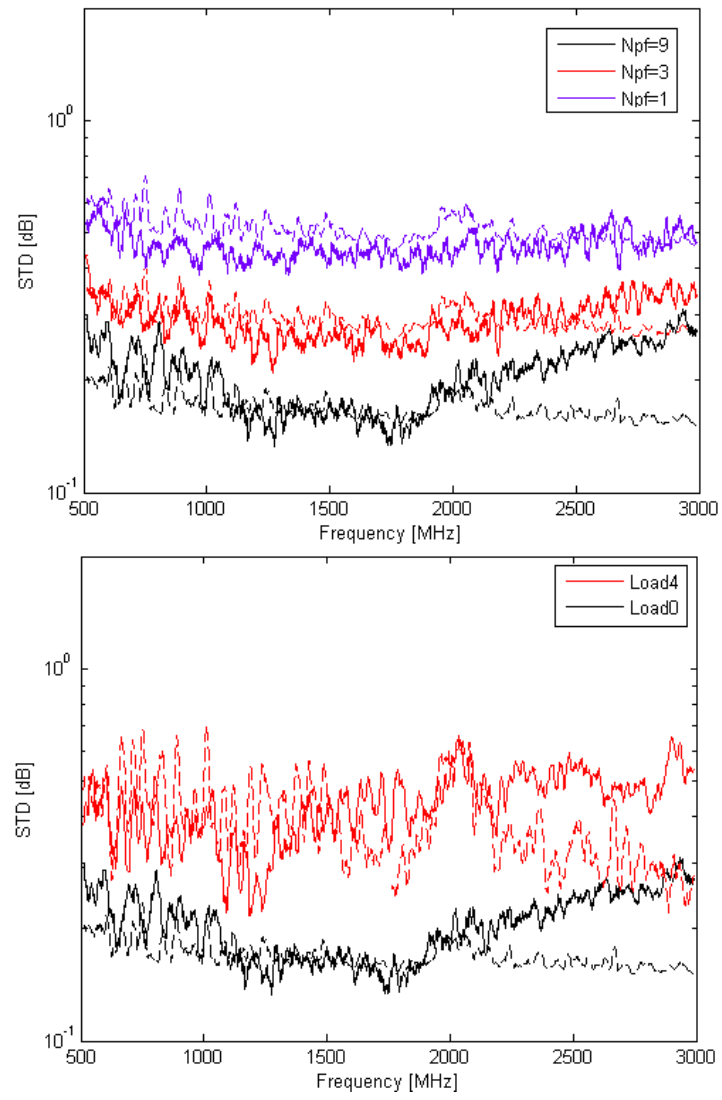

Fig. 1. Uncertainty measured in the reverberation chamber at NIST, Boulder. Upper graph: in an unloaded chamber and using platform stirring with 1, 3 and 9 positions averaged respectively. Lower graph: nine platform positions are used and curves are presented for two different loading cases.

[2] P.-S. Kildal and K. Rosengren, "Correlation and capacity of MIMO systems and mutual coupling, radiation efficiency and diversity gain of their antennas: Simulations and measurements in reverberation chamber", IEEE Communications Magazine, vol. 42, no. 12 , pp. 102-112, Dec. 2004.

[3] D. A. Hill, M. T. Ma, A. R. Ondrejka, B. F. Riddle, M. L. Crawford, and R. T. Johnk, "Aperture excitation of electrically large, lossy cavities", Electromagnetic Compatibility, IEEE Transactions on, vol. 36, pp. 169-178, 1994

[4] J.G. Kostas, B. Boverie, "Statistical model for a mode-stirred chamber",IEEE Transactions on Electromagnetic Compatibility, Vol. 33, pp. 366-370, 1991

[5] P.-S. Kildal, S.-H. Lai, and X. Chen, "Direct Coupling as a Residual Error Contribution During OTA Measurements of Wireless Devices in Reverberation Chamber", IEEE AP-S International Symposium on Antennas and Propagation, Charleston, USA, June 1-5, 2009

[6] P.-S. Kildal, X. Chen, C. Orlenius, M. Franzén, C. Lötbäck Patané, "Characterization of Reverberation Chambers for OTA Measurements of Wireless Devices: Formulation of Channel Matrix and Uncertainty", submitted to IEEE Transactions on Antennas and Propagation, Sept. 2011

[7] X. Chen, P.-S. Kildal, S.-H. Lai, "Estimation of average Rician $\mathrm{K}$-factor and average mode bandwidth in loaded reverberation chamber", IEEE Antennas and Wireless Propagation Letters, Vol. 10, pp. 1437-1440, Dec. 2011

[8] X. Chen, P.-S. Kildal, J. Carlsson, J. Yang, "Comparison of Ergodic Capacities from Wideband MIMO Antenna Measurements in Reverberation Chamber and Anechoic Chamber", Antennas and Wireless Propagation Letters, vol. 10, pp. 446-449, May 2011 